\title{
Progress on the Development of Future Airport Surface Wireless Communications Network
}

\author{
Robert J. Kerczewski \\ NASA Glenn Research Center \\ Cleveland, Ohio, USA \\ $+12164333434$ \\ rkerczewski@nasa.gov ${ }^{12}$ \\ James M. Budinger \\ NASA Glenn Research Center \\ Cleveland, Ohio, USA \\ $+12164333496$ \\ james.m.budinger@nasa.gov
}

\author{
David E. Brooks \\ Morgan Franklin \\ Cleveland, Ohio, USA \\ dbrooks@grc.nasa.gov \\ Steve DeHart \\ Syracuse, New York, USA
}

steve.dehart@sensis.com

\author{
Robert P. Dimond \\ Verizon Business \\ Cleveland, Ohio, USA \\ bdimond@grc.nasa.gov \\ Michael Borden \\ Syracuse, New York, USA \\ mike.borden@sensis.com
}

Abstract - Continuing advances in airport surface management and improvements in airport surface safety are required to enable future growth in air traffic throughout the airspace, as airport arrival and departure delays create a major system bottleneck. These airport management and safety advances will be built upon improved communications, navigation, surveillance, and weather sensing, creating an information environment supporting system automation. The efficient movement of the digital data generated from these systems requires an underlying communications network infrastructure to connect data sources with the intended users with the required quality of service. Current airport surface communications consists primarily of buried copper or fiber cable. Safety related communications with mobile airport surface assets occurs over $25 \mathrm{kHz}$ VHF voice and data channels. The available VHF spectrum, already congested in many areas, will be insufficient to support future data traffic requirements. Therefore, a broadband wireless airport surface communications network is considered a requirement for the future airport component of the air transportation system. Progress has been made on defining the technology and frequency spectrum for the airport surface wireless communications network. The development of a test and demonstration facility and the definition of required testing

${ }^{1}$ U.S. Government work not protected by U.S. copyright.

${ }^{2}$ IEEEAC paper \#1493, Version 6, Updated 12 January, 2008. and standards development are now underway. This paper will review the progress and planned future work.

\section{TABLE OF ConTents}

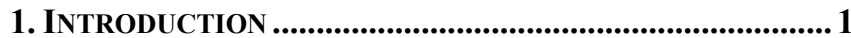

2. COMMUNICATIONS TECHNOLOGY .................................... 2

3. OPERATING SPECTRUM ................................................... 3

4. NeXTGEN CNS TESTBED ................................................. 3

5. TEST PROGRAM ............................................................6 6

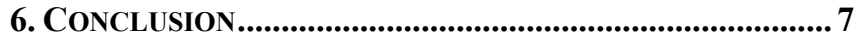

REFERENCES........................................................................... 7

BIOGRAPHIES................................................................. 7

\section{INTRODUCTION}

Next generation air transportation systems will achieve high levels of efficiency and safety through a combination of new air traffic management methods, automation, and collaboration. The underlying information infrastructure that will support the transformation from current to future systems requires major increases in communications capacity and performance.

The Eurocontrol-FAA Future Communications Study has confirmed that a single communications system connecting the aircraft to the air traffic management system is not 
feasible. Due to physical and operational differences in the different flight domains - airport, terminal area, en-route, and oceanic/remote/polar - a combination of several types of communications links is required. For the airport surface, the study recommended a wireless communications network based on the IEEE 802.16e [1]. The operating frequency being considered is $5091-5150 \mathrm{MHz}$.

In order to enable a safe and reliable airport surface communications network that is interoperable between airports both nationally and globally, a development and test program followed by completion of technical standards will be undertaken. For this purpose, a test facility known as the NextGen CNS Testbed has been developed at the Cleveland Hopkins International Airport and the adjacent NASA Glenn Research Center (GRC). The testbed will include a prototype airport surface wireless communications network based on the IEEE 802.16e standard, and will enable system tests and demonstrations leading to definition of an airport surface wireless communications network standard.

The following sections will provide a description of the progress on airport wireless communications network development to date, the NextGen CNS Testbed and its airport surface communications test infrastructure, review technical results and future test plans.

\section{COMmunications TECHNOLOGY}

For the airport surface, results of previous studies by the NASA GRC indicating the optimal type of communications system were confirmed by the Future Communications Study. An airport surface communication system based on industry communications was desired to minimize cost and take advantage of on-going improvements, as opposed to the more costly development of a proprietary standard which reaches obsolescence before the system is fully deployed. Occupying the "Microwave Landing System
(MLS) Extension Band" of 5091-5150 MHz, a wireless mobile communications network based on the IEEE 802.16 e standard was selected as the optimum technology to be developed and introduced in the future.

The 802.16e-based system is envisioned as a multi-node network wireless network covering all areas of the airport surface and, if necessary, areas beyond the airport proper where facilities and equipment such as lighting, navigational aids, weather sensors and wake sensors might need to be located. The system would accommodate all mobile communications requirements including aircraft, various types of ground vehicles, and personnel, and also enable links to fixed airport assets. Aircraft that are parked at a gate or taxiing would also be able to access the system, as long as the aircraft is not in flight.

The planned airport surface network will be scalable for small, medium and large airports through the use of one or more network nodes, or cells. It will also be interconnected with existing wired network infrastructure to create a hybrid network communications capability with enhanced reliability. For airports without existing wired network infrastructure, the wireless communications network system infrastructure can be installed much more easily and at far lower cost than wired infrastructure when such communications network capability is required.

Table 1 summarizes some of the key features of several IEEE 802.16 versions. The $802.16 \mathrm{e}$ version is the technology selected for the airport surface because it provides several key capabilities. It can maintain connection with moving vehicles at speeds up to $120 \mathrm{~km} / \mathrm{hr}$ so that taxiing aircraft as well as other airport surface vehicles can enter the network. It can work with mobile users in non-line-of-sight (NLOS) conditions, an essential element for an airport surface environment with many large moving aircraft. It can provide up to $50 \mathrm{Mbps}$ in the 2-6 $\mathrm{GHz}$ band, which matches the available spectrum as described below, in cells of radius 1-3 miles appropriate for

\begin{tabular}{|l|l|l|l|}
\hline & $802.16 / \mathrm{c}$ & $\mathbf{8 0 2 . 1 6 a / R E V d} / 2004$ & $802.16 \mathrm{e}$ \\
\hline Spectrum & $11-66 \mathrm{GHz}$ & $2-11 \mathrm{GHz}$ & $2-6 \mathrm{GHz}$ \\
\hline $\begin{array}{l}\text { Channel } \\
\text { Conditions }\end{array}$ & LOS & LOS, NLOS & NLOS \\
\hline Bit Rate & $32-124 \mathrm{Mbps}$ & $1-70 \mathrm{Mbps}$ & Up to 50 Mbps \\
\hline Modulation & $\begin{array}{l}\text { QPSK, 16QAM } \\
\text { and 64QAM }\end{array}$ & $\begin{array}{l}\text { OFDM 256 sub-carriers, QPSK, } \\
\text { 16QAM and 64QAM }\end{array}$ & $\begin{array}{l}\text { OFDMA, QPSK, 16QAM and } \\
64 Q A M\end{array}$ \\
\hline Mobility & Fixed & Fixed, Portable & Mobile (upto 120Km/h) \\
\hline $\begin{array}{l}\text { Channel } \\
\text { Bandwidths }\end{array}$ & $\begin{array}{l}20,25 \text { and 28 } \\
\text { MHz }\end{array}$ & $\begin{array}{l}\text { Selectable channel bandwidths } \\
\text { between } 1.5 \text { and } 20 \mathrm{MHz}\end{array}$ & $\begin{array}{l}\text { Selectable channel bandwidths } \\
\text { between } 1.25 \text { and } 20 \mathrm{MHz}\end{array}$ \\
\hline $\begin{array}{l}\text { Typical Cell } \\
\text { Radius }\end{array}$ & $1-3$ miles & $\begin{array}{l}\text { 3-5 miles } \\
\text { Maximum range } 30 \mathrm{miles} \text { based } \\
\text { on the tower height }\end{array}$ & $1-3$ miles \\
\hline
\end{tabular}


a mesh network architecture on an airport surface. The $802.16 \mathrm{e}$ standard also has a number of other desirable features including: Orthogonal Frequency Division Multiple Access (OFDMA) with variable power, spreading and reuse; support of a full range of smart antenna technologies such as beamforming, space-time code, and spatial multiplexing; support of hard-handoff, fast base station switching and macro diversity handover; and support of multicast and broadcast service. Adaptive modulation and coding, using QPSK, 16QAM and 64QAM, and convolutional and convolutional turbo code with variable code rate are among the 802.16e options which enable adaptation to link conditions to maintain a high QoS. Multiple device/user authentication, flexible key management protocol, strong traffic encryption, control and management plane message protection and security protocol optimizations for fast handovers are available to provide the necessary data and network security.

\section{OPERATING SPECTRUM}

Studies by NASA GRC, Mitre CAASD, and others have defined a spectrum requirement for airport surface wireless communications in the range of 60 to $100 \mathrm{MHz}[2,3]$. One spectrum band considered was the MLS Extension Band, 5091-5150 MHz. This aviation band was previously a navigation band reserved for continuing the deployment of microwave landing systems when the core MLS band reached capacity. However, the MLS system deployment has occurred at a much lower rate than expected, so the unused extension band became vulnerable to other industrial users. At the same time, saturation of the VHF band for aeronautical communications required that other bands for future aeronautical communications be identified.

The 2007 World Radiocommunications Conference (WRC) included an agenda item to consider additional allocations for aeronautical mobile (route) service (AMS(R)S) in order to allow for additional bandwidth for aeronautical communications. The agenda item proposed to first look at existing aeronautical bands to see if $A M(R) S$ allocations could be added in those bands. The MLS Extension Band was one of the aeronautical bands under consideration for such an allocation. However, this band also includes a coprimary allocation for mobile satellite service (MSS) uplinks, hence only low power communications links could be introduced into this band without causing interference to the existing MSS systems.

An airport surface wireless communication network, which would require of short-range communications links of 2-5 miles, was thus considered ideal for the MLS Extension Band. An $\operatorname{AM}(\mathrm{R}) \mathrm{S}$ allocation in this band, limited in scope to airport surface applications, was proposed. This allocation was supported by a number of studies by NASA, FAA, Mitre CAASD [4], and others that indicated such a system could operate in the 5091-5150 MHz band without interfering with the MSS uplinks. In addition, the available
$59 \mathrm{MHz}$ of bandwidth fulfills the majority of the expected spectrum requirement.

A proposal to allocate the $5000-5030 \mathrm{MHz}$ band also for $\mathrm{AM}(\mathrm{R}) \mathrm{S}$ for the airport surface application was postponed and will be considered again at the 2011 WRC. An additional $30 \mathrm{MHz}$ of bandwidth would provide a total of $89 \mathrm{MHz}$ for the airport surface wireless communications network or $89 \%$ of the expected total requirement.

\section{NeXtGen CNS TeSTBED}

In order to maintain this new allocation, progress on the development and demonstration of the future airport surface wireless network needs to be shown. At the same time, eventual implementation of these systems requires the national and international standards processes to be initiated. The identification and validation of a specific $802.16 \mathrm{e}$ "aviation profile", which would form the basis of standards, requires research, modeling and testing.

Next Generation Air Transportation System (NextGen) Communication, Navigation and Surveillance (CNS) Test Bed has resulted from a cooperative agreement between the Sensis Corporation and NASA Glenn Research Center (GRC) [5]. The test bed focuses on the evaluation of promising CNS technologies and systems, presently encompasses three airports in the Cleveland, Ohio region and a Test and Demonstration Center located at GRC. The test bed surveillance coverage area extends to include a 200 mile radius around the Cleveland metropolitan area thus creating a wide area air-ground test and demonstration capability. The three included airports (Hopkins International, Burke Lakefront, and Lorain County Regional) are representative of various classes of airports across the country close to population centers that will be central to accommodating forecasted air travel growth over the next 20 years.

Sensis, in partnership with the Cleveland Airport System, the Lorain County Regional Airport Authority, and the FAA, has installed an advanced version of their multilateration surveillance system and wireless communications infrastructure at each of these airports. Implemented as well is a prototype of a Remote Tower System (a.k.a., a Staffed Virtual Tower) that "shadow controls" operations at Burke Lakefront Airport from the Test and Demonstration Center some 13 miles away. The Test Bed is integrated as a system via a prototype of a regional information sharing and management system.

Sensis, GRC, and their Test Bed partners are collaborating to define and test possible solutions for some of the most significant challenges to the success of NextGen. In addition to remote monitoring and control of airports, the issues under investigation by this team include validating the promised efficiencies of negotiated 4-D arrival and departure trajectories, assessing advanced integrated 
surveillance capabilities, helping solve the continuing safety problem of runway incursions, and improving the efficiency of airport and airline surface operations.

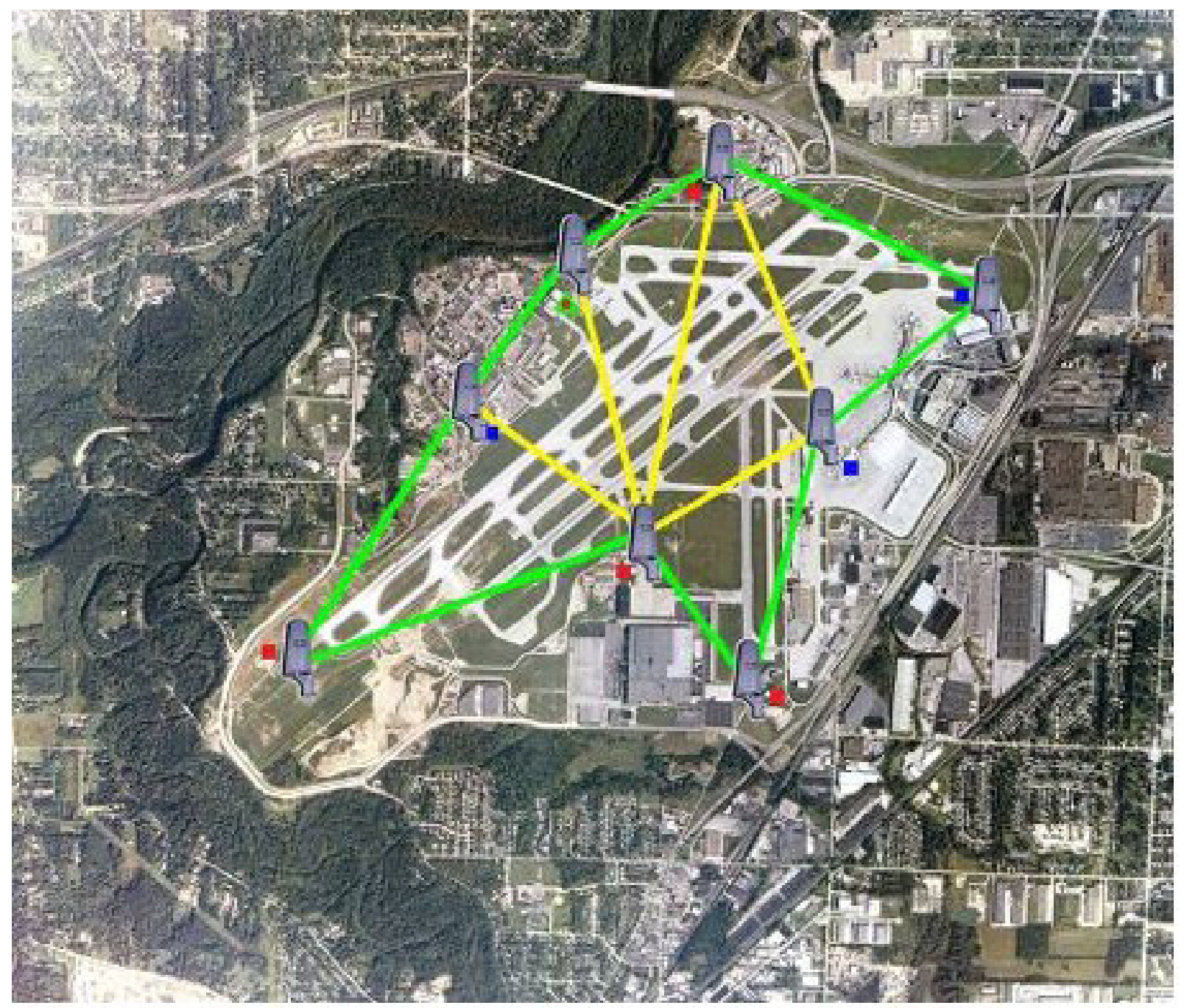

Figure 1 - NextGen CNS Testbed wireless communications network node locations at the Cleveland Hopkins International Airport.

The initial implementation of airport surface communications capability has focused on wireless solutions suitable for the secure movement of aviation surveillance data and the remote monitoring and maintenance of the wireless communications nodes. A primary motivation for pursuing a wireless solution is to overcome the installation and maintenance costs associated with trenched, hard line implementations.

A $5 \mathrm{GHz}$ wireless mesh network is implemented as the current backbone network for the Test Bed. Figure 1 shows the locations of the wireless communications network nodes, which are co-located with the Sensis multilateration system equipment. The current network is implemented in an unlicensed portion of the $5 \mathrm{GHz}$ band. This experimental network transports the multilateration surveillance sensor data and system commands between the central target processor and the remote multilateration sensor units. This network is implemented with commercial off-the-shelf wireless network nodes that are based on the 802.11a wireless standard, but with enhanced capabilities. These enhancements include advanced mesh networking and a sectored antenna design that increases link performance and improves interference rejection. The nodes are modified to operate in either the unlicensed 5 $\mathrm{GHz}$ middle and upper U-NII bands, or the FAA controlled (but currently unused) Extended MLS band (5.091-5.150 $\mathrm{GHz}$ ). The nodes can also operate at reduced RF signal bandwidths, including 5 and $10 \mathrm{MHz}$ to optimize spectrum efficiency.

A second network is implemented in the $900 \mathrm{MHz}$ ISM band to support remote configuration, control, and maintenance of the remote sensor and communications sites 
located at each airport.

The wireless system currently implemented has a multitude of characteristics that can be examined. Because of its mesh topography implemented in an environment with dynamic LOS blockage and multi-path reflection events, zero-state mesh convergence, full state mesh reconvergence times are currently being examined.

Table 2 - MAC Layer statistics for Skygateway 2 indicating peer, frequency, Remote Signal Strength Indication (RSSI) and corresponding raw data rates.

\begin{tabular}{|l|lcccc|}
\hline \multicolumn{5}{|c|}{ Active Path Links to SkyGateway2 } \\
Freq & Peer & Local & Remote & $\begin{array}{c}\text { Local } \\
\text { TxMod }\end{array}$ & $\begin{array}{c}\text { Remote } \\
\text { (MHz) }\end{array}$ \\
Tode & RSSI & RSSI & (Mbps) & (Mbps) \\
\hline 5150 & Ext7 & 37 & 40 & 54 & 54 \\
5150 & Ext8 & 35 & 38 & 54 & 54 \\
5150 & Ext14 & 35 & 35 & 48 & 36 \\
5150 & Ext15 & 40 & 36 & 54 & 54 \\
\hline 5200 & Ext7 & 38 & 42 & 48 & 48 \\
5200 & Ext8 & 35 & 37 & 48 & 54 \\
5200 & Ext14 & 34 & 33 & 48 & 54 \\
5200 & Ext15 & 40 & 36 & 54 & 54 \\
\hline 5250 & Ext7 & 36 & 40 & 54 & 54 \\
5250 & Ext8 & 37 & 35 & 54 & 54 \\
5250 & Ext14 & 34 & 34 & 48 & 48 \\
5250 & Ext15 & 38 & 34 & 48 & 36 \\
\hline 5300 & Ext7 & 38 & 42 & 48 & 54 \\
5300 & Ext8 & 35 & 39 & 54 & 54 \\
5300 & Ext14 & 33 & 35 & 54 & 54 \\
5300 & Ext15 & 37 & 35 & 54 & 54 \\
\hline 5320 & Ext7 & 37 & 47 & 48 & 54 \\
5320 & Ext8 & 38 & 40 & 54 & 54 \\
5320 & Ext14 & 33 & 38 & 54 & 54 \\
5320 & Ext15 & 35 & 36 & 54 & 54 \\
\hline
\end{tabular}

Table 3 - Characterization of link performance for bandwidth, jitter, and loss during line rate UDP transfers of varying payload data lengths.

\begin{tabular}{|l|l|l|l|l|l|}
\hline \multicolumn{6}{|c|}{ IP statistics between SkyExtender8 to SkyGateway2 } \\
\hline $\begin{array}{l}\text { Data } \\
\text { (Bytes) }\end{array}$ & $\begin{array}{l}\text { Max BW } \\
(\mathrm{Mbps})\end{array}$ & $\begin{array}{l}\text { Jitter } \\
(\mathrm{ms})\end{array}$ & Lost & Total & $\begin{array}{l}\% \\
\text { Lost }\end{array}$ \\
\hline 100 & 3.000 & 0.833 & 140 & 112153 & 0.125 \\
\hline 300 & 8.250 & 0.214 & 479 & 103503 & 0.463 \\
\hline 500 & 13.200 & 0.811 & 75 & 99076 & 0.076 \\
\hline 700 & 15.900 & 0.449 & 12148 & 97549 & 12.45 \\
\hline 1000 & 16.600 & 0.944 & 12616 & 74977 & 16.82 \\
\hline 1300 & 16.900 & 0.767 & 9030 & 57692 & 15.65 \\
\hline 1470 & 16.600 & 0.907 & 8634 & 51019 & 16.92 \\
\hline
\end{tabular}

Characterization of the network's proprietary MAC layer performance, as well as the network's IP data rate, jitter, and loss characteristics has been completed by Mike Borden of the Sensis Corporation using Iperf, a common network analysis tool. Tables $3 \& 3$ illustrate sample MAC and IP layer data obtained from the conducted tests.

The communications system design for an airport surface must take into account the unique channel characteristics of the airport environment at the frequency of operation, in particular the highly dynamic multipath environment of an airport, where large cylindrical reflecting elements are in constant motion at varying speeds throughout the area.

The characterization effort was carried out by Ohio University, in cooperation with the Federal Aviation Administration (FAA) and NASA GRC. It was composed of analysis, computer simulations of channel dynamics and field measurement components. The analysis phase used previous research work completed on terrestrial mobile and satellite communications to bound channel parameters and included the development of a channel classification scheme. Development of detailed channel simulation models includes the effects of channel dispersion and fading, and the variation of these characteristics over time and space. Field measurements included the collection of a comprehensive set of measurement data.

Channel measurements were accomplished by a process labeled "sounding", a field activity involving the transmission and reception of a known test signal. The signal used in airport channel measurement campaigns was a direct sequence spread spectrum with known correlation properties with a bandwidth of $50 \mathrm{MHz}$ and transmit power of 2 W. [6]

The measurements were carried out at several US airports of different sizes and configurations. Measurements at Cleveland, Miami and JFK New York airports gathered data relevant to large and medium size airports. Measurements were also made at Tamiami and Burke Lakefront airports to represent small airport facilities. The measurements were made at various locations in and around airport grounds. The following are some of the conclusions from the measurement campaign.

For bandwidths above $1 \mathrm{MHz}$, the airport surface is very dispersive and for successful modeling over a typical communications channel a statistically non-stationary tapped delay line model with detailed tap amplitude statistics and pairwise tap correlation coefficients is required [7]. It was observed that, in some instances, fading was severe with amplitude statistics characterized as being worse than Rayleigh. Furthermore, multipath components were identified to have random "birth-death" like behavior in time.

The airport surface can be categorized into three distinct 
propagation regions: least dispersive line of sight open, intermediate non-line of sight specular and more dispersive non-line of sight. Employing airport sites located inside the airport fence can enhance signal strength for areas that are not line of sight.

The final evaluation indicates that a communications system for airport applications operating in the $5091-5150 \mathrm{MHz}$ band can meet the communications requirements for the airport surface domain. This result was further clarified by work performed by ITT Inc. for NASA under the Future Communications Study. In this work, ITT used a channel model adapted from a detailed model developed from the results of the Ohio University channel measurements to create an approximation of the airport propagation channel at $5.1 \mathrm{GHz}$. Models of the 802.16e transmitter and receiver were developed and evaluated against the airport channel model [8]. The results of the simulations indicated good performance for the IEEE 802.16e standard in the airport environment in the 5091-5150 MHz band.

As mentioned above, the NextGen CNS Testbed wireless network is currently implemented via IEEE 802.11 technology. The next step to support the development, validation and standardization of the airport surface wireless communications network requires the transition to $802.16 \mathrm{e}$ equipment, operating in the MLS extension bands. This transition has been planned and requires the acquisition of 802.16e equipment operating at the required frequencies. Such equipment is expected to become available in the second quarter of 2009. The initial test program to be executed when this equipment has been installed is described in the following section.

\section{Test Program}

Once the IEEE 802.16e equipment has been installed within the NextGen CNS Testbed, system characterization test are planned, followed by application demonstrations. The application demonstrations are in the early stages of being defined, but will include such applications as surveillance data collection, weather sensor data collection, status and control of navigational aids, and mobile asset tracking.

System characterization tests consist of three categories: development of system parameters, handoff mechanisms to mitigate blockage and enable mobility, and path loss and interference characterization.

The development of system parameters requires testing of RF link performance, including signal strength and communications coverage (both line of sight and non-line of sight) at all airport locations, as well as measurement of message integrity, communications latency, and message rate and data throughput. These parameters will be measured as a function of range, network loading, and quality of service (QoS) requested.
Another set of measurements will enable the determination of system parameters required to ensure that the airport surface network will not interfere with MSS satellite uplink, a precondition of using the MLS extension band. Measurements of transmit power vs. coverage, message integrity, latency, rate and throughput will be used to assess the performance of the system in conditions that maintain non-interference with the MSS uplinks.

In order to develop a methodology for channelization of the MLS extension band, testing of message rate, data throughout and base station coverage, as a function of the surface wireless communications network architecture and physical configuration, base station coverage capability and frequency re-use plan will be employed.

Alternative handoff mechanisms will be evaluated for their ability to enable mobility among the airport surface network nodes and mitigate temporary blockage due to aircraft and ground vehicle movement. Testing of the ability to maintain connectivity, handoff transition times, and message integrity, latency, rate and throughput will be performed for both the mobility case (moving between base station cells) and the temporary blockage case, while considering network data load equalization and other factors.

Mobility testing will be accommodated by using a ground mobile laboratory known as the Terrestrial Hybrid Environment for Verification of Aeronautical Networks (THEVAN). THEVAN has performed a variety of mobile network testing with both ground and satellite-based systems [9] and will be configured as an 802.16e mobile node to enable testing of the mobility parameters described above. THEVAN will enable measurement of signal blockage events and corresponding network reconfiguration times, correlating these events with the type of physical blockage encountered through video recording of line-ofsight. Both simulated blockages and real-world blockages resulting from the relative motion of THEVAN, representing a network-connecting vehicle, and actual moving aircraft on the airport surface will be included in the testing.

Path loss and interference characterization are additional factors leading to optimum system design and band channelization methodologies. The propagation path loss exponent, path loss standard deviation, and shadow margin will be measured as a function of antenna height, antenna pattern and gain for both line of sight and non-line of sight conditions.

It is expected that additional testing requirements will be identified during the planning, installation, test and demonstration phases of the program. 


\section{Conclusion}

The requirement for an advanced airport surface communications network has been established through NASA studies, as well as through air transportation system planning efforts such as NextGen and SESAR. Although communications networks based on buried copper and fiber-optic cable are available in a number of airports, they are found to be expensive to install and maintain and are vulnerable to cuts, resulting in service loss. A wireless communications infrastructure can add robustness and reliability to an airport communications network at a much lower cost and can be configured to reach all areas within and adjacent to the airport surface. Studies including the Eurocontrol-FAA Future Communications Study have identified the IEEE 802.16e standard as the commercial standard preferred for am airport surface wireless communications network.

Frequency spectrum has been identified in the MLS Extension Band (5091-5150 MHz) that is well suited for an airport surface wireless application that meets a majority of the bandwidth requirement. Characterization of this frequency band at several operational airports has been completed, and has led to the development of channel models. Simulations based on these models indicates that good performance of the IEEE 802.16e standard in an airport environment in the MLS Extension Band can be expected.

The NextGen CNS Testbed has been developed at Cleveland Hopkins International Airport and two smaller nearby airports. The testbed is based upon multilateration surveillance equipment installed by Sensis and an airport surface wireless communications network. The current network is based upon 802.11e equipment, however the installation of $802.16 \mathrm{e}$ equipment operating within the MLS Extension Band is imminent. The $802.16 \mathrm{E}$ equipment will make possible a range of system test which will enable the development and validation of an 802.16e "aviation profile" that will become a blueprint for a national and international standard for airport surface wireless communicators networks. The testbed thus equipped will then be able to test and demonstrate a wide range of current and future applications that will support the advances in airport surface management and improvements in airport surface safety required to enable the future growth in air traffic envisioned by NextGen and SESAR.

\section{REFERENCES}

[1] Robert J. Kerczewski,, James M. Budinger, and Tricia J. Gilbert, "Technology Assessment Results of the Eurocontrol/FAA Future Communications Study", 2008 IEEE Aerospace Conference, March, 2008.

\section{BIOGRAPHIES}

[2] Rafael D. Apaza, "Wireless Communications for Airport Surface: An Evaluation of Requirements", 2005 IEEE Aerospace Conference, March, 2005.

[3] Izabela Gheorghisor, "Spectral Requirements of ANLE Networks for the Airport Surface," Mitre Report MP080109R1, July, 2008.

[4] Izabela Gheorghisor, Yan-Shek Hoh, and Frank Box, "Compatibility Analysis of Airport Wireless Local Area Networks and Satellite Feeder Links in the 5091-5150 MHz Band," 2006 ICNS Conference, May, 2006.

[5] Dana Hall and James M. Budinger, "NextGen ATS Communications, Navigation, and Surveillance Test Bed", $26^{\text {th }}$ Digital Avionics System Conference, October, 2007.

[6] David W. Matolak, Lawrence R. Foore, and Rafael D. Apaza, "Channel Characterization in the $5 \mathrm{GHz}$ Microwave Landing System Extension Band for Future Airport Surface Communications", $5^{\text {th }}$ ICNA Conference and Workshop, May, 2005.

[7] "Wireless Channel Characterization in the $5 \mathrm{GHz}$ Microwave Landing System Extension Band for Airport Surface Areas", Final Project Report for NASA ACAST Project, Ohio University, May 2006.

[8] Glen Dyer, Tricia J. Gilbert, and James M. Budinger, "FCS Phase II Results Paper 3 - Detailed Technology Investigations", ICAO Aeronautical Communications Panel Working Group C Meeting \#11, Working Paper 9, September, 2006.

[9] David Brooks, Ryan Wilkins, and Douglas Hoder, "Mobile IP Communications for Aeronautical Applications", $23^{\text {rd }}$ Digital Avionics System Conference, October, 2004. 


\section{BIOGRAPHIES}

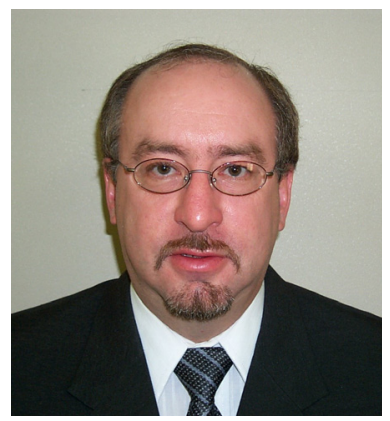

Robert J. Kerczewski has been involved with research and development of satellite communications systems and applications since for the Analex Corporation (19821986) and NASA (1986present). He holds a BEE degree from Cleveland State University (1982) and an MSEE degree from Case Western Reserve University (1987). He is currently the Project Manager for the NASA's Advanced CNS Architectures and System Technologies (ACAST) Project.

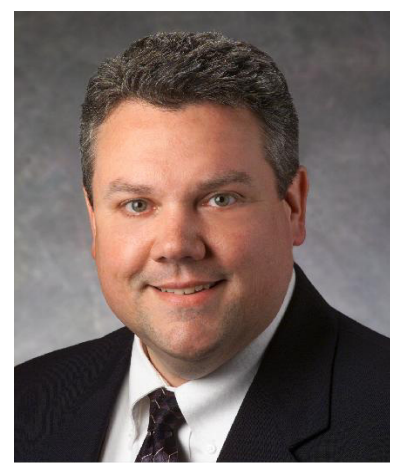

James. M. Budinger is a senior engineer in the Communications Systems Integration Branch at NASA Glenn Research Center (GRC) in Cleveland Ohio. Over nearly 30 years of service at NASA, Mr. Budinger has lead research and advanced technology development for both space and aeronautical communications as a researcher, supervisor, and technology project manager. He holds MSEE and BEE degrees from Cleveland State University. Mr. Budinger received a NASA Exceptional Service Medal in 2003 for outstanding project and technical achievements in the development of advanced communications technology. Most recently, he led NASA's technology assessment for the Eurocontrol/FAA-NASA Future Communications Study to determine an internationally harmonized solution for the next generation of aeronautical voice and data communications. Currently, he leads GRC's research in airport surface wireless communications via a cooperative agreement with Sensis Corporation for the NextGen CNS Test Bed at GRC and three nearby regional airports.
David E. Brooks has over 14 years of advanced IP networking and advanced RF communication system integration experience on various projects at Glenn

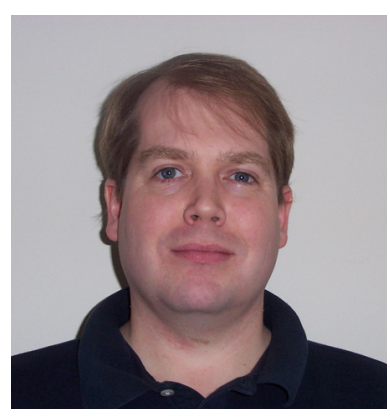

Research Center at Lewis Field as a Support Service Contractor(SSC) working for Sterling Software, Inc (1994-1999), Infinite Global Infrastructures, LLC (1999-2006) and MorganFranklin, Corporation

Present). These projects have included Advanced Communication Technology Satellite(ACTS) 622 Mbps high data

terminal experiments, Ku-band networking link experiments using mobile and air assets under Advanced Air Transportation Technologies(AATT) and supporting wireless/wired airport surface NexGen research activities. He holds a BEE degree from Cleveland State University (1992) and a MSEE degree from Cleveland State University (1997).

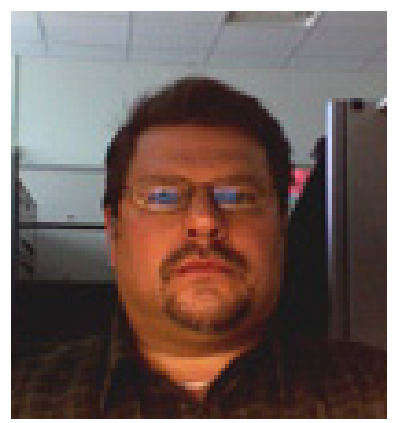

Robert P. Dimond is currently employed by Verizon Business working on several projects at NASA Glenn Research Center in Cleveland Ohio. His career spans nearly twenty years involving the research, implementation, and troubleshooting of network protocols and applications within satellite, mobile, and aeronautical environments. 